Relations industrielles

Industrial Relations

\title{
L'obligation de paix du travail - étude du droit suisse et comparé, par Gabriel Aubert, Genève, Librairie de l'Université, Georg et Cie S.A., 1981, 306 pp., ISBN 2-8257-0078-9.
}

\section{Pierre Verge}

Volume 38, numéro 2, 1983

URI : https://id.erudit.org/iderudit/029365ar

DOI : https://doi.org/10.7202/029365ar

Aller au sommaire du numéro

Éditeur(s)

Département des relations industrielles de l'Université Laval

ISSN

0034-379X (imprimé)

1703-8138 (numérique)

Découvrir la revue

Citer ce compte rendu

Verge, P. (1983). Compte rendu de [L'obligation de paix du travail - étude du droit suisse et comparé, par Gabriel Aubert, Genève, Librairie de l'Université, Georg et Cie S.A., 1981, 306 pp., ISBN 2-8257-0078-9.] Relations industrielles / Industrial Relations, 38(2), 442-444. https://doi.org/10.7202/029365ar

Tous droits réservés @ Département des relations industrielles de l'Université Laval, 1983
Ce document est protégé par la loi sur le droit d'auteur. L'utilisation des services d'Érudit (y compris la reproduction) est assujettie à sa politique d'utilisation que vous pouvez consulter en ligne.

https://apropos.erudit.org/fr/usagers/politique-dutilisation/ 
Il s'agit d'un ouvrage de bonne qualité et à propos duquel on ne peut que regretter qu'il n'intègre pas la Charte canadienne des droits et libertés de la personne. Néanmoins, le texte n'en perd pas pour autant son intérêt. Bien au contraire car il permet dans une certaine mesure de vérifier l'à-propos et les limites de la Charte.

L'étude est avant tout descriptive et accorde peu de place à la discussion fondamentale. L'analyse est menée avec beaucoup de rigueur et apparaît fort complète. Les références sont nombreuses. L'auteur a divisé ses propos en quatre parties mais fondamentalement le texte est constitué par trois axes principaux.

Le premier axe est d'ordre historique et se retrouve dans la première partie (4 chapitres). Cette partie est la plus intéressante même s'il ne faut pas y rechercher une étude en profondeur des situations de discrimination à diverses époques. La présentation en est plutôt une de mise en contexte et d'évolution. Le lecteur peut apprendre comment la discrimination s'est manifestée sur le territoire canadien à divers moments de notre histoire. Il est intéressant de lire ce que l'on a généralement appris de bouche à oreilles, du bout des lèvres. Cette partie démontre que le Canada a connu et connaît ses heures de discrimination.

Les deuxième et troisième parties forment le second axe de l'ouvrage. Neuf chapitres permettent de se familiariser avec les sujets et matières susceptibles de fonder la discrimination dans diverses formes d'activités. Ce volet du volume présente avant tout un intérêt en ce qu'il permet l'acquisition de connaissances sinon techniques à tout le moins pratiques. L'auteur n'a rien ménagé pour offrir un tableau d'ensemble qui n'est pas trop chargé tout en étant suffisamment élaboré. La comparaison entre les encadrements législatifs des diverses provinces permet de dégager ce qui pourrait être un cadre de référence pour une étude analytique et critique d'une pensée canadienne sur la discrimination.
Finalement, le dernier axe se préoccupe de la description des organismes administrateurs et des voies de recours. On retrouve cette présentation dans les deux chapitres de la partie quatrième.

Discrimination and the Law est un ouvrage de références qui peut être avantageusement utilisé comme manuel d'enseignement pratique ou comme source de renseignements.

\section{Rodrigue BLOUIN}

\section{Université Laval}

\section{L'obligation de paix du travail - étude du} droit suisse et comparé, par Gabriel Aubert, Genève, Librairie de l'Université, Georg et Cie S.A., 1981, 306 pp., ISBN 2-8257-0078-9

L'auteur s'interroge en profondeur sur l'«effet pacificateur» de la convention collective, sur l'interdiction qui pourrait en résulter, pour ses signataires et ceux qu'elle lie, de recourir pendant sa durée aux moyens de pression, essentiellement la grève et le lockout, soit pour modifier ou compléter l'accord, soit pour en assurer la fidèle exécution. Plus spécifiquement, il se propose de cerner la position à cet égard de son système national, le droit suisse, objet de la seconde partie de l'ouvrage, en le comparant à certains systèmes juridiques étrangers, soit ceux de la République fédérale d'Allemagne, de la Suède, des États-Unis, du Royaume-Uni, de la France et de l'Italie, à l'analyse desquels il aura consacré la première moitié du volume.

Il s'agit là - le lecteur sent le besoin d'exprimer d'emblée sa réaction d'ensemble - d'une entreprise comparatiste d'envergure, portant sur les aspects fondamentaux de ces différents systèmes de relations collectives de travail. Elle s'exprime dans un texte dense, mais très structuré, qui confronte les grands courants jurisprudentiels et doctrinaux des différents pays en cause. On examine tour à tour la source de cette obligation de paix, 
dans la mesure où elle s'affirme dans un système national, son contenu, c'est-à-dire les comportements qu'elle prescrit ou défend, son objet, à savoir les matières au sujet desquelles doivent correspondre les comportements indiqués. On précise également les «sujets» de cette obligation, soit le cercle des personnes ou des groupements visés et, enfin, les sanctions auxquelles son inobservation peut donner lieu.

Pour ce qui est de la source de l'obligation de paix, l'auteur se demande dans quelle mesure celle-ci peut découler, d'une part, du contenu normatif - ou substantif - de l'accord collectif, d'autre part, de l'établissement de procédures destinées à résoudre les litiges du travail, soit dans l'entreprise, soit même à un niveau national, lorsqu'il s'agit de systèmes comportant la conclusion d'accords de cette envergure.

La nature de la convention collective est en cause: ne s'agit-il que du simple constat du rapport de forces entre les parties, de la solution momentannée d'un conflit susceptible de renaître à tout moment? La convention collective revêt-elle, au contraire, une véritable dimension contractuelle, se présente-t-elle comme un instrument de stabilisation des relations professionnelles? Des systèmes, qui, tels les systèmes français et italiens, se rattachent à la première conception, verront dans la grève un moyen de lutte sociale auquel la conclusion d'un accord collectif ne saurait faire obstacle. Partant, l'obligation de paix, si d'exception elle existe, ne pourra être que le résultat d'un accord exprès; elle n'est point «immanente à la convention». De même en est-il, en principe toujours, au Royaume-Uni, la convention collective se situant dans l'ajuridique, à la manière d'un gentlemen's agreement. En revanche, en Allemagne la doctrine et la jurisprudence, d'une façon prépondérante, adoptent des vues "structuralistes»: l'obligation de paix n'a pas à être volontairement et expressément assumée; elle découle de la nature même de la convention collective. Elle lui est, a-t-on dit, immanente. Elle correspond aux effets directs et impératifs de la convention collective sur les contrats indi- viduels de travail; elle est la contrepartie des conditions de travail assumées par le patronat: d'un point de vue synallagmatique, pacta sunt servanda. La Suède et la Suisse explicitent législativement une telle solution, non étrangère dans ce dernier cas, à l'influence de la doctrine germanique prépondérante. Aux États-Unis, deux dispositions de la loi fédérale du travail consacrent la portée "contractuelle» de l'accord collectif: l'une, l'article 8 (d) du National Labor Relations Act, avant tout reliée au processus même de la négociation collective, exclut en principe du devoir de négocier la discussion ou l'acceptation d'une modification des termes et conditions de travail contenus dans une convention de durée déterminée; l'autre, primordiale, l'article 301 (a) de la même loi, donne ouverture à l'action civile pour violation du contrat et assure ainsi la sanction de la clause usuelle d'interdiction de la grève et du lock-out pendant la durée de la convention et l'effet juridique de la clause d'arbitrage.

L'expérience américaine en témoigne: l'obligation de paix peut ne pas trouver uniquement sa source dans les conditions substantives de travail. Elle peut provenir, d'une façon complémentaire ou non, de l'établissement de procédures permettant aux travailleurs ou à leurs représentants de porter devant des instances appropriées des conflits pouvant survenir à la suite de l'accord. De telles dispositions font aussi partie de l'échange entre les signataires de la convention, du quid pro quo. Il peut s'agir alors notamment de l'arbitrage de tels conflits, comme dans les systèmes américains et suisse, pays où la pratique de clauses expresses de paix absolue ou intégrale pendant la durée de la convention est répandue. L'auteur fait remarquer toutefois qu'en Suisse de tels engagements ont, plus souvent qu'aux États-Unis, pour contrepartie l'arbitrage des conflits d'intérêts pendant la convention, en plus, bien entendu de celui auquel peut donner lieu son contenu. Et le vocabulaire quelque peu martial, correspondant forcément à l'ensemble du sujet, de laisser ici place à la constatation suivante au sujet du système suisse (p. 181): «(...) les rap- 
ports entre les parties ressemblent alors à ceux qui caractérisent le contrat de société»

Il serait injuste pour l'auteur de tenter d'évoquer en si peu de mots le contenu fort dense des autres chapitres que nous avons annoncés: non seulement ceux où, précisément, l'on discute ainsi de l'étendue, relative ou absolue, de l'obligation de paix, mais aussi ces pages où il est question de son contenu: d'un point de vue négatif, prohibe-t-on uniquement les "mesures de combat» usuelles que sont la grève et le lock-out? La prohibition s'étend-elle également aux contraintes pouvant s'exercer sur des tiers par voie de boycottage? Notons en passant à cet égard que, si la République fédérale et la Suède définissent largement l'acte préjudiciable visé, la Suisse va jusqu'à y inclure l'atteinte à la réputation de la partie adverse. Positivement cette fois, jusqu'où va le devoir d'un signataire d'agir sur ceux qu'il représente - groupements intermédiaires, le cas échéant et travailleurs individuels - pour qu'ils respectent l'obligation de paix?

Cette question particulière met en cause le type de négociations pouvant avoir cours dans les différents pays, selon qu'elle se pratique par ensembles sectoriels nationaux, ou qu'elle se ramène le plus souvent à des accords d'entreprises, comme aux États-Unis. Il en va de même de la détermination des sujets de l'obligation de paix, au-delà des signataires de l'accord: le problème de l'assujettissement des instances syndicales intermédiaires ne sera pas constant. Quant aux salariés euxmêmes, le rôle variable que pourra jouer le contrat individuel de travail témoignera aussi de la diversité, proprement juridique cette fois, des systèmes en cause. Cette diversité se manifestera, enfin dans la nature des sanctions de l'obligation de paix, dans les formes de l'intervention de type judiciaire auquel elle peut donner lieu: souci assez généralisé, peuton dire, de rétablir avant tout l'«autorité de la convention».

Certes, la réalité de la représentativité syndicale, celle de la pratique de grève également - l'ampleur du mouvement, son orien- tation - varient considérablement de certains de ces systèmes que l'auteur compare, aux autres. Néanmoins, grâce aux divers angles d'analyse juridique proposés, il devient possible d'apprécier globalement l'intensité de cette obligation de paix du travail dans les différents pays retenus: elle émerge avec force en Allemagne, en Suède, aux États-Unis et, particulièrement, en Suisse. Cette vision d'ensemble peu commune à laquelle $\mathrm{M}$. le docteur Aubert nous permet d'avoir accès est le résultat de la confrontation constante, rigoureuse et nuancée, des grands cheminements doctrinaux et jurisprudentiels suivis dans les pays dont il s'agit.

Pierre VERGE

Université Laval

Les mesures disciplinaires: étude jurisprudentielle et doctrinale, par C. D'Aoust, L. Leclerc et G. Trudeau, Montréal, École de relations industrielles, Monographie 13, 1982, 484 pp., ISBN 2-89067-012-0, ISSN 0708-9945

Cet ouvrage se situe dans le prolongement de la monographie titrée «La jurisprudence arbitrale québécoise en matière de congédiement». Deux (2) des auteurs du présent volume avaient d'ailleurs signé la monographie: D'Aoust et Leclerc.

La somme de travail exigée pour finaliser ce précis sur les mesures disciplinaires apparaît colossale: plus de mille cinq cents (1500) décisions analysées en outre de nombreuses références doctrinales. Comparativement à la monographie antérieure le nombre de décisions a pratiquement triplé. Le lecteur trouvera donc dans le nouveau texte une documentation plus que complémentaire.

La première partie du volume est consacrée principalement à l'élaboration du modèle conceptuel retenu de la discipline industrielle, perspective juridique. On peut notamment y lire une description des principales obligations et fautes corrélatives du salarié 